\title{
Hypoxia-induced responses by endothelial colony-forming cells are modulated by placental growth factor
}

\author{
Michelle B. Hookham', Imran H. A. Ali ${ }^{1}$, Christina L. O'Neill', Emer Hackett ${ }^{1}$, Melanie H. Lambe', Tina Schmidt ${ }^{1}$, \\ Reinhold J. Medina', Sara Chamney², Bharathi Rao ${ }^{3}$, Eibhlin McLoone ${ }^{2}$, David Sweet ${ }^{3}$, Alan W. Stitt ${ }^{1}$ \\ and Derek P. Brazil ${ }^{1 *}$
}

\begin{abstract}
Background: Endothelial colony-forming cells (ECFCs), also termed late outgrowth endothelial cells, are a welldefined circulating endothelial progenitor cell type with an established role in vascular repair. ECFCs have clear potential for cell therapy to treat ischaemic disease, although the precise mechanism(s) underlying their response to hypoxia remains ill-defined.

Methods: In this study, we isolated ECFCs from umbilical cord blood and cultured them on collagen. We defined the response of ECFCs to $1 \% \mathrm{O}_{2}$ exposure at acute and chronic time points.

Results: In response to low oxygen, changes in ECFC cell shape, proliferation, size and cytoskeleton phenotype were detected. An increase in the number of senescent ECFCs also occurred as a result of long-term culture in 1\% $\mathrm{O}_{2}$. Low oxygen exposure altered ECFC migration and tube formation in Matrige ${ }^{\circledast}$. Increases in angiogenic factors secreted from ECFCs exposed to hypoxia were also detected, in particular, after treatment with placental growth factor (PIGF). Exposure of cells to agents that stabilise hypoxia-inducible factors such as dimethyloxalylglycine (DMOG) also increased PIGF levels. Conditioned medium from both hypoxia-treated and DMOG-treated cells inhibited ECFC tube formation. This effect was reversed by the addition of PIGF neutralising antibody to the conditioned medium, confirming the direct role of PIGF in this effect.
\end{abstract}

Conclusions: This study deepens our understanding of the response of ECFCS to hypoxia and also identifies a novel and important role for PIGF in regulating the vasculogenic potential of ECFCs.

Keywords: Hypoxia, Endothelial progenitor cells, Endothelial colony-forming cells, Placental growth factor, Senescence, Migration

\section{Background}

Since the first description of endothelial progenitor cells (EPCs) in 1997 as bone marrow-derived vascular precursors with reparative potential [1], there has been considerable interest in their utility for cell-based therapy of ischaemic vasculopathies. "EPC" refers to a broad range of vascular progenitors characterised using diverse cell surface markers and functional endpoints. Endothelial colony-forming cells (ECFCs), also known as late EPCs or

\footnotetext{
*Correspondence: d.brazil@qub.ac.uk

${ }^{1}$ Centre for Experimental Medicine, Queen's University Belfast, 97 Lisburn

Road, Belfast BT9 7BL, UK

Full list of author information is available at the end of the article
}

outgrowth endothelial cells (OECs), are a subset of circulating EPCs with the ability to differentiate into endothelial cells and mediate vascular repair [2]. When isolated, ECFCs have significantly higher proliferative capacity compared with mature endothelial cells, and they maintain their endothelial progenitor phenotype during long-term ex-vivo expansion [3]. ECFCs are also capable of supporting vascular repair in vivo by integration into pre-existing vasculature, significantly reducing areas of ischaemia in murine models of retinopathy, myocardial infarction and stroke [4]. 
ECFCs have potential for use as cell-based therapy to treat ischaemic diseases by aiding vascular repair as well as establishing new vasculature [5]. However, the function and number of ECFCs and other EPC subtypes have been reported to be impaired in a range of vascular disease states such as diabetes [6,7], cardiovascular disease [8], preeclampsia [9] and vascular development in premature infants [10]. The number and function of ECFCs has also been shown to be impaired in low birth weight infants [11], a patient cohort at risk of retinal ischaemia in infancy and cardiovascular disease in adulthood. Accelerated senescence in premature ECFCs as a result of decreased SIRT1 expression was also demonstrated recently [12]. Therefore, it is important to understand what happens when ECFCs enter a hypoxic environment and what molecular cues are required to stimulate ECFC-mediated vascular repair.

Placental growth factor (PlGF) is a member of the vascular endothelial growth factor (VEGF) family that has been demonstrated to promote angiogenesis both independently and synergistically via heterodimer formation with VEGF-A to activate Flt1/VEGF receptor-1 signalling [13]. PlGF also binds to the neuropilin-1/ VEGF165 receptor and stimulates angiogenesis [14]. Since its discovery in 1991 [15], PlGF has been associated with a number of disease states [16] including ischaemia [17], cardiovascular disease [18], cancer [19], arthritis [20] and preeclampsia [21]. Recent reports have revealed that there is increased secretion of PlGF from ECFCs isolated from end-stage renal failure patients [22] while in-vitro exposure to PIGF can enhance tubulogenic function in ECFCs [18]. These findings suggest that PlGF may have an important if ill-defined role in normal ECFC vasoreparative function. The signalling and functional responses of ECFCs exposed to PlGF remains to be elucidated, especially in the context of vascular insufficiency and hypoxia encountered by cells as they home to areas of localised tissue damage [23]. In this study, we define the effects of acute and chronic hypoxia on ECFCs in the absence of other confounding factors. In response to low oxygen, we observed changes in ECFC cell size, shape, cytoskeletal structure and proliferative potential. Low oxygen altered ECFC scratch wound repair and in-vitro tube formation in Matrigel $^{\circ}$. Of particular interest, we observed increased levels of PIGF secreted from ECFCs exposed to hypoxia and dimethyloxalylglycine (DMOG), a HIF stabilising agent. Conditioned medium from these cells inhibited tube formation in fresh ECFCs. This effect was reversed by neutralising antibodies against PIGF, confirming its central role in this effect. Improved knowledge of how ECFCs respond to low oxygen in vitro will aid understanding of how they may adapt their function to aid vascular repair in ischaemic diseases.

\section{Methods}

ECFC isolation, in-vitro culture and hypoxia exposure ECFCs were isolated from umbilical cord blood of fullterm neonates. Mononuclear cells (MNCs) were obtained by density gradient fractionation, re-suspended in complete medium (EGM-2; Lonza) supplemented with $10 \%$ FBS and seeded on 24-well culture plates precoated with rat tail collagen type 1 (BD Biosciences) at a density of $1 \times 10^{7}$ cells $/ \mathrm{ml}$. Colonies typically appeared within 2-3 weeks, and ECFC clones were typically cultured for $>20$ passages. A humidified, temperaturecontrolled hypoxia chamber (Coy Laboratories) was used to maintain cells in $1 \% \mathrm{O}_{2}$. Culture media were preconditioned to $1 \% \mathrm{O}_{2}$ prior to use to ensure instantaneous hypoxia exposure and the temperature was maintained at $37{ }^{\circ} \mathrm{C}$. A Casy cell counter-analyser (Roche) was used for cell counts, viability and cellular size assessment.

\section{Immunocytochemistry}

Cells were fixed in $4 \%(\mathrm{w} / \mathrm{v})$ paraformaldehyde for $20 \mathrm{~min}$ at room temperature. Following PBS washes, cells were permeabilised with $0.1 \%$ Triton X-100 for 10 min at RT and then blocked in $1 \%$ bovine serum albumin (BSA) for $2 \mathrm{~h}$ in PBS before overnight incubation in primary antibody at $4{ }^{\circ} \mathrm{C}$. After washing with PBS, cells were incubated with appropriate secondary antibody for $1 \mathrm{~h}$ at RT and imaged under a confocal fluorescence scanning microscope (Nikon). Primary antibodies phalloidin (Invitrogen) and vinculin (Sigma-Aldrich) were used to stain F-actin and focal adhesion complexes respectively. Anti-mouse Alexa Fluor 568 IgG (Invitrogen) was used as secondary antibody. Mounting medium contained the nuclear stain DAPI (Vectashield; Vector Laboratories).

\section{Flow cytometry}

For staining, $5 \times 10^{5}$ ECFCs were used per sample. Cells were filtered using cell strainers with a $35-\mu$ m nylon mesh (BD Falcon; BD Biosciences), re-suspended in $100 \mu \mathrm{l}$ PBS buffer and incubated with respective antibodies for $45 \mathrm{~min}$ at $4{ }^{\circ} \mathrm{C}$. Antibodies reactive to $\mathrm{CD} 31$ (platelet endothelial cell adhesion molecule (PECAM)), CD45 (protein tyrosine phosphatase receptor type C), CD14 (monocyte differentiation antigen; eBioscience) and CD146 (melanoma cell adhesion molecule; BD Biosciences) were utilised. After staining, cells were washed in PBS and finally resuspended in $1 \mathrm{ml}$ PBS for analysis using an Attune Acoustic Focusing Flow Cytometer (Invitrogen Life Technologies). At least 20,000 events were acquired for each sample. Respective isotype controls were used to determine accurate settings for data analysis.

\section{Protein extraction and western blotting}

Protein was extracted from ECFCs using ice-cold RIPA containing protease and phosphatase inhibitors 
as described previously. Twenty micrograms of protein was resolved by electrophoresis through $10 \%$ SDS polyacrylamide gel and electro-transferred onto PVDF membrane (Millipore). The membranes were blocked for $1 \mathrm{~h}$ at RT with 3\% (w/v) non-fat dry milk in Tris-buffered saline with $0.1 \%$ Tween-20 (TBS-T). After blocking, the membranes were incubated overnight at $4{ }^{\circ} \mathrm{C}$ with primary antibodies reactive to HIF- $1 \alpha$ (\#610958; BD Biosciences) and HIF-2 $\alpha$ (R\&D Systems). After three washes with TBS-T, the membranes were incubated for $1 \mathrm{~h}$ with their respective HRP-conjugated secondary antibodies (Santa Cruz). Three TBS-T washes were carried out before the protein bands were detected using ECL (Merck Millipore). Membranes were re-probed with $\beta$-actin (Cell Signaling Technology) as a housekeeping gene to ensure equal loading.

\section{Scratch wound repair assay}

ECFCs were grown on collagen-coated 24-well plates until confluent. The monolayer was then scratched using a p200 pipette tip to create a wound. After gentle washing with PBS, the growth media was changed to serum-free EGM-2. The scratched area was imaged using ImageJ analysis software, and the wound distance was measured at the beginning $\left(\mathrm{T}_{0}\right)$ and end of the experiment $\left(\mathrm{T}_{\mathrm{X}}\right)$. The following formula was used to convert the migrated area of the scratch wound into a percentage:

$$
\% \text { of wound closure }=T_{0}-T_{X}=X / T_{0} * 100 .
$$

\section{Cell viability}

ECFC viability was determined using the Cell Counting Kit-8 (CCK-8; Sigma) as per the manufacturer's instructions. Cells were seeded in 96-well collagen-coated plates $\left(1 \times 10^{4}\right.$ cells/well $)$. Cells were treated for $24 \mathrm{~h}$ with $100 \mathrm{ng} / \mathrm{ml}$ human recombinant PlGF (R\&D Systems) or vehicle (4 mM HCl 0.1\% BSA). Absorbance was measured at $450 \mathrm{~nm}$ using an Omega plate reader.

\section{Proteome profiling}

A proteome profiler human angiogenesis array $(R \& D$ Systems) was used to analyse the secretion of angiogenic cytokines from ECFCs. Cells were maintained in serumfree medium in 21 and $1 \% \mathrm{O}_{2}$ for $24 \mathrm{~h}$, in accordance with the manufacturer's instructions. ImageJ image analysis software was used to calculate densitometry and all results were normalised to a positive control. PlGF levels in cell culture supernatant were measured using a PlGFspecific ELISA (R\&D Systems) according to the manufacturer's instructions.

\section{DMOG treatment}

ECFCs were grown in complete media (EGM-2; Lonza) and treated with either dimethylsulfoxide (DMSO, vehicle) or $1 \mathrm{mM}$ DMOG (Sigma-Aldrich) for $24 \mathrm{~h}$. At harvest, media were removed and stored at $-80{ }^{\circ} \mathrm{C}$ for analysis, after which the cells were washed in ice-cold PBS prior to further analysis by western blot analysis. PlGF levels in cell culture supernatant were measured using a PlGF-specific ELISA (R\&D Systems) according to the manufacturer's instructions.

\section{Tubulogenesis assay}

ECFCs were labelled using a fluorescence membrane labelling kit (PKH; Sigma) or Calcein AM dye (Molecular Probes) according to the manufacturer's protocol. Between $8 \times 10^{4}$ and $1 \times 10^{5}$ labelled ECFCs were resuspended in basement membrane matrix (Matrigel ${ }^{\circ}$; BD Biosciences). Fifty-microlitre aliquots were spotted onto four-well plates. After polymerisation, the spots were covered in endothelial cell growth medium (EGM-2; Lonza) or a 1:1 ratio of EGM-2 and conditioned media. Wells were assessed for the presence of tube-like structures after 24, 48 and $72 \mathrm{~h}$, and were imaged using a confocal scanning microscope (Nikon). For PlGF neutralising experiments, ECFCs $\left(7.5 \times 10^{5}\right.$ cells) were mixed with conditioned medium collected from previously treated ECFCs exposed to vehicle or DMOG (1 mM) and resuspended in basement membrane Matrix (Matrigel $\left.{ }^{\oplus}\right)$. Next, 50- $\mu$ l aliquots were spotted onto a 24-well plate and allowed to polymerise. After polymerisation, spots were covered in conditioned medium from vehicle or DMOG-treated cells in the presence of a PlGF neutralising antibody (MAB264; R\&D Systems) or an isotype IgG control (Sigma). Matrigel ${ }^{\bullet}$ spots were labelled with Calcein AM dye (Molecular Probes) prior to imaging. Wells were assessed for the presence of tube-like structures after $24 \mathrm{~h}$, and imaged using a confocal scanning microscope (Nikon). The vascular tube area was quantified using NIS Elements software (Nikon).

\section{Reverse-transcription and real-time PCR}

Total RNA was isolated using an RNeasy Mini Kit (Qiagen). cDNA was synthesised from 500 ng RNA using Superscript III (Invitrogen). Semi-quantitative real-time PCR was performed using gene-specific Real Time ready Custom Single assays for ANGPTL4, SLC2A1 and housekeeping genes $\beta$-actin and 18S (Roche). Relative quantitative values were obtained using the $\Delta \Delta \mathrm{Ct}$ method.

\section{$\beta$-Galactosidase activity}

ECFCs were grown on collagen-coated glass coverslips prior to senescence analysis. $\beta$-Galactosidase activity was detected using the Senescence $\beta$-galactosidase Staining 


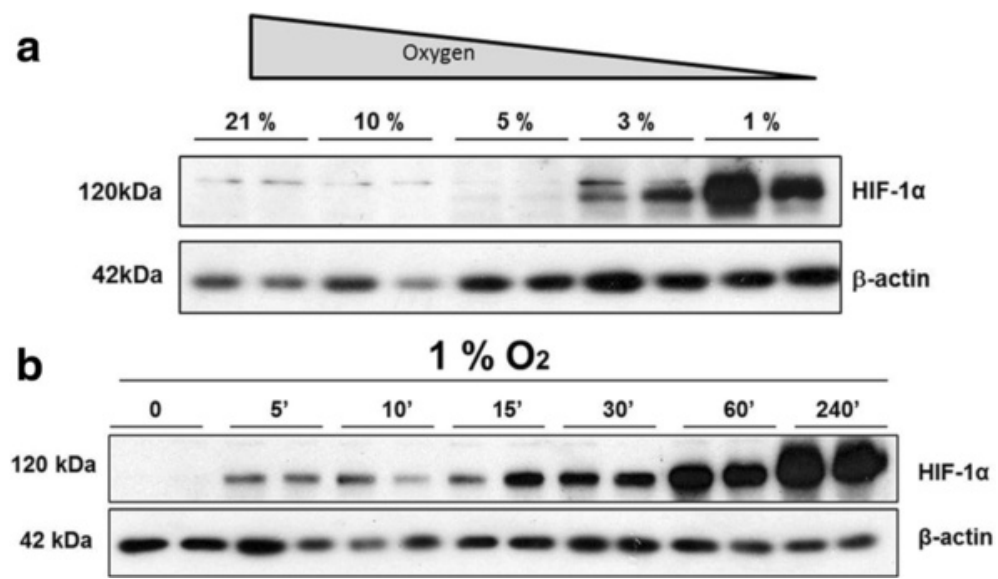

Fig. 1 HIF-1a stabilisation in ECFCS occurs rapidly at 1\% $\mathrm{O}_{2}$. ECFCs were grown in 12\% FCS-supplemented EBM-2 medium. a ECFCS were cultured at the indicated oxygen tension for 5 h. Protein lysates were probed using HIF-1a (top panel), with $\beta$-actin included as a loading control (bottom panel). b ECFCs were cultured in full EBM medium at $1 \% \mathrm{O}_{2}$ for the indicated time range, starting at 5 min (5) and ending at 240 min (240). Cells were lysed and protein lysates were probed with HIF-1a (top panel), with $\beta$-actin included as a loading control (bottom panel) ( $n=3$ ). HIF-1a hypoxia inducible factor 1 alpha
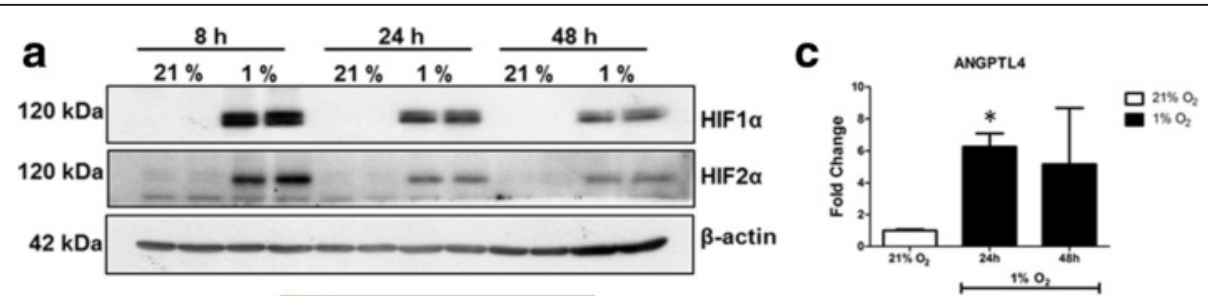

b
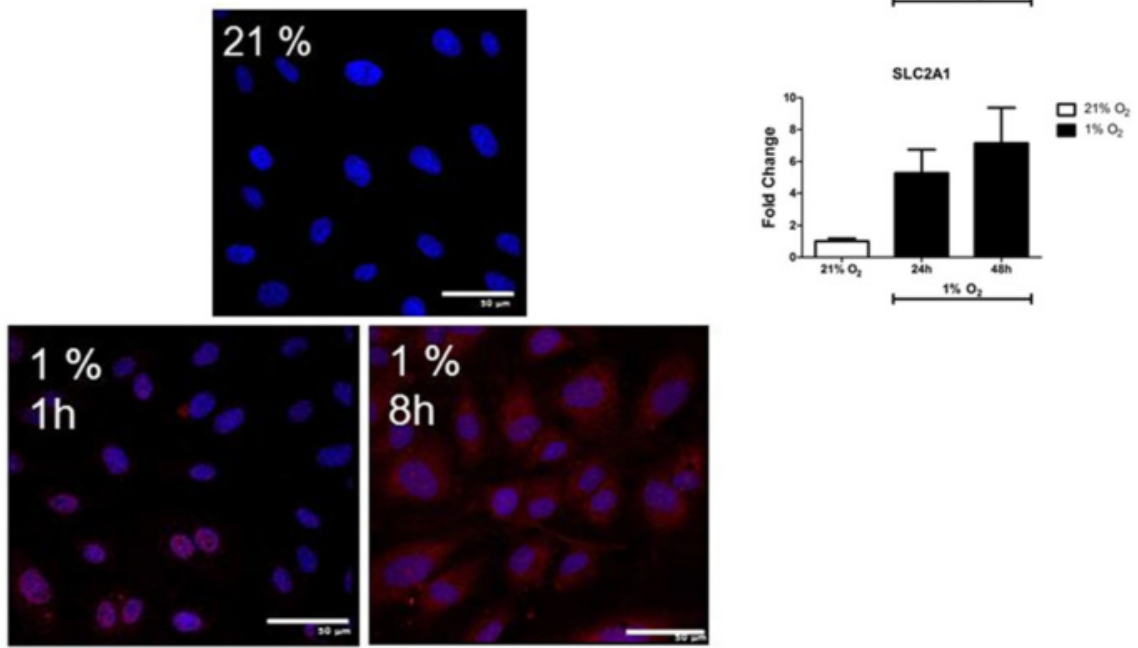

Fig. 2 ECFC responses to $1 \% \mathrm{O}_{2}$. ECFCs were grown in 12\% FCS-supplemented EBM-2 medium and maintained in either 21 or $1 \% \mathrm{O}_{2}$ for the indicated times. a Protein lysates isolated from time-matched ECFCs grown under normal conditions $\left(21 \% \mathrm{O}_{2}\right)$ or hypoxic conditions $\left(1 \% \mathrm{O}_{2}\right)$ were probed with HIF-1a (top panel) or HIF-2a, with $\beta$-actin included as a loading control (bottom panel). b. ECFCs cultured at 21 or $1 \%$ for $5 \mathrm{~h}$ were permeabilised and incubated with antibodies reactive to HIF-a (red) and DAPI to stain the nuclei (blue). Scale bar: 50 HM. c Real-time PCR was carried out on cDNA generated from ECFCs incubated in $1 \% \mathrm{O}_{2}$ for the indicated times. Levels of HIF-1 responsive genes ANGPTL4 and SLC2A1 were measured, and normalised to the mean of two housekeeping genes (18S and $\beta$-actin). The $\triangle \triangle C t$ subtractive method was used to compare between treatments, with $21 \% \mathrm{O}_{2}$ as the calibrator. Fold-changes compared with $21 \% \mathrm{O}_{2}$ were calculated. Data plotted as mean foldchange \pm SD. ${ }^{*} p<0.05(n=5)$. HIF-1a hypoxia inducible factor 1 alpha (Colour figure online) 
Kit (Cell Signalling Technology) according to the manufacturer's instructions. The cell monolayer was imaged using a phase contrast microscope (Nikon). The percentage of positively stained cells was quantified using ImageJ image analysis software.

\section{Statistical analysis}

Data were analysed using Student's two-tailed paired or unpaired $t$ test or ANOVA and were plotted as mean \pm standard deviation (SD) unless otherwise indicated.

\section{Results}

ECFCs were isolated from umbilical cord blood of normal term infants as described previously [24]. The endothelial character of these cells was confirmed by the expression of PECAM (CD31) and melanoma metastasis-associated surface molecule (MUC18, CD146) (Additional file 1: Figure S1). In addition, isolated ECFCs did not express the leukocyte markers CD45 and the myeloid marker CD14, which confirmed that these cells were not hematopoietic in nature (Additional file 1: Figure S1). To examine the effects of low oxygen on these cells, ECFCs were grown in a range of oxygen tensions from $21 \%$ (atmospheric oxygen, $760 \mathrm{mmHg}$ ) to $10,5,3$ and $1 \%(36 \mathrm{mmHg}$, Fig. 1a). HIF$1 \alpha$ immunoreactivity was only detected at $\leq 3 \% \mathrm{O}_{2}$, with the highest levels of HIF- $1 \alpha$ detected at $1 \%$ (Fig. 1a). The inhibition of prolyl-hydroxylases and accumulation of cytoplasmic HIF- $1 \alpha$ at $1 \% \mathrm{O}_{2}$ was extremely rapid, and was detected within 5 min of hypoxia exposure (Fig. 1b).
A time-dependent increase in HIF-1 $\alpha$ accumulation occurred in ECFCs, with the strongest accumulation present after $4 \mathrm{~h}$ (240 $\mathrm{min}$ ) of hypoxia exposure. Based on these data, all subsequent hypoxia exposure experiments were carried out at $1 \% \mathrm{O}_{2}$ for a minimum of $4-5 \mathrm{~h}$.

Accumulation of both HIF- $1 \alpha$ and HIF- $2 \alpha$ isoforms were observed in ECFCs grown in $1 \% \mathrm{O}_{2}$ for 8,24 and $48 \mathrm{~h}$ (Fig. 2a). In comparison with ECFCs grown in $21 \%$ $\mathrm{O}_{2}(160 \mathrm{mmHg})$, cells grown in $1 \% \mathrm{O}_{2}$ demonstrated a marked increase in HIF-1 $\alpha$ immunoreactivity in the cytosol, with punctate staining in the nuclei evident at the $5 \mathrm{~h}$ time point (Fig. 2b). This suggested that the HIF-1 $\alpha$ subunit was translocating to the nucleus as part of the typical transcriptional response to hypoxia [25]. This was further corroborated by the increased expression of HIF- $1 \alpha$ regulated transcripts ANGPTL4 and SLC2A1 (also known as GLUT1) in ECFCs grown in $1 \% \mathrm{O}_{2}$ (Fig. 2c). Thus, exposure to low oxygen concentrations induces a typical "hypoxia" response in terms of HIF- $1 \alpha$ transcription factor accumulation and gene transcription in ECFCs.

ECFC exposure to hypoxia induced the formation of actin stress fibres, with cells displaying a more organised, linear arrangement of F-actin (Fig. 3ii, v). Vincullin staining also revealed the appearance of focal adhesions in ECFCs grown in $1 \% \mathrm{O}_{2}$, at the terminal ends of the striated F-actin fibres (Fig. 3vi). These data suggest that low oxygen concentrations triggered cytoskeletal changes in ECFCs suggestive of cellular stress and an adaption to the hypoxic environment by strengthening their attachment
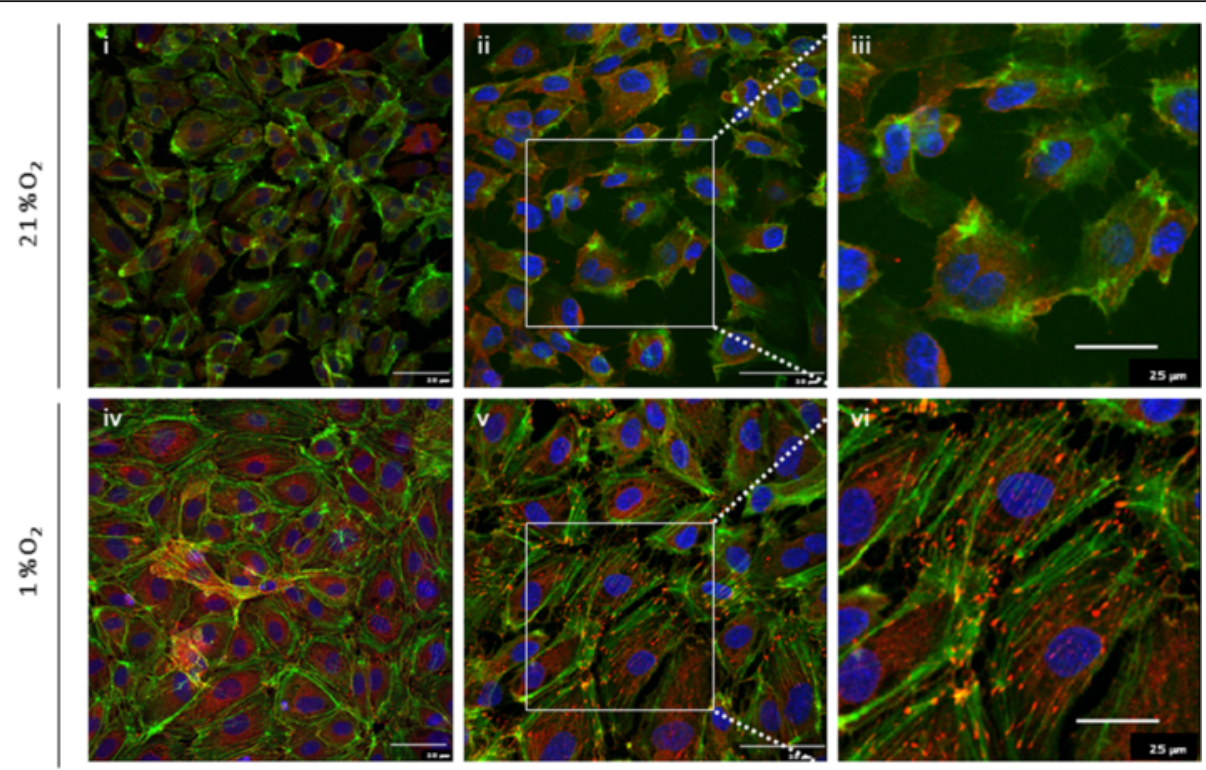

Fig. 3 Hypoxia induces actin rearrangement and stress fibre formation in ECFCs. The ECFCs were grown in 12\% FCS-supplemented EBM-2 medium and maintained in either 21 or $1 \% \mathrm{O}_{2}$ for $24 \mathrm{~h}$. ECFCs were stained with phallodin to identify filamentous actin (F-actin, green), vincullin (red) to identify focal adhesions and DAPI to stain the nuclei (blue). i, iv $40 \times$ magnification, ii, $v 60 \times$ magnification. i, ii, iv, $v$ Scale bars: $50 \mu \mathrm{m}$ size marker. ii, $v$ Magnified area depicted in iii and vi. Actin stress fibres (green) and focal adhesions (red) are evident in a single cell grown in $1 \% \mathrm{O}_{2}$ (iii, vi). iii, vi Scale bar: $25 \mu \mathrm{m}$ size marker $(n=3)$ 


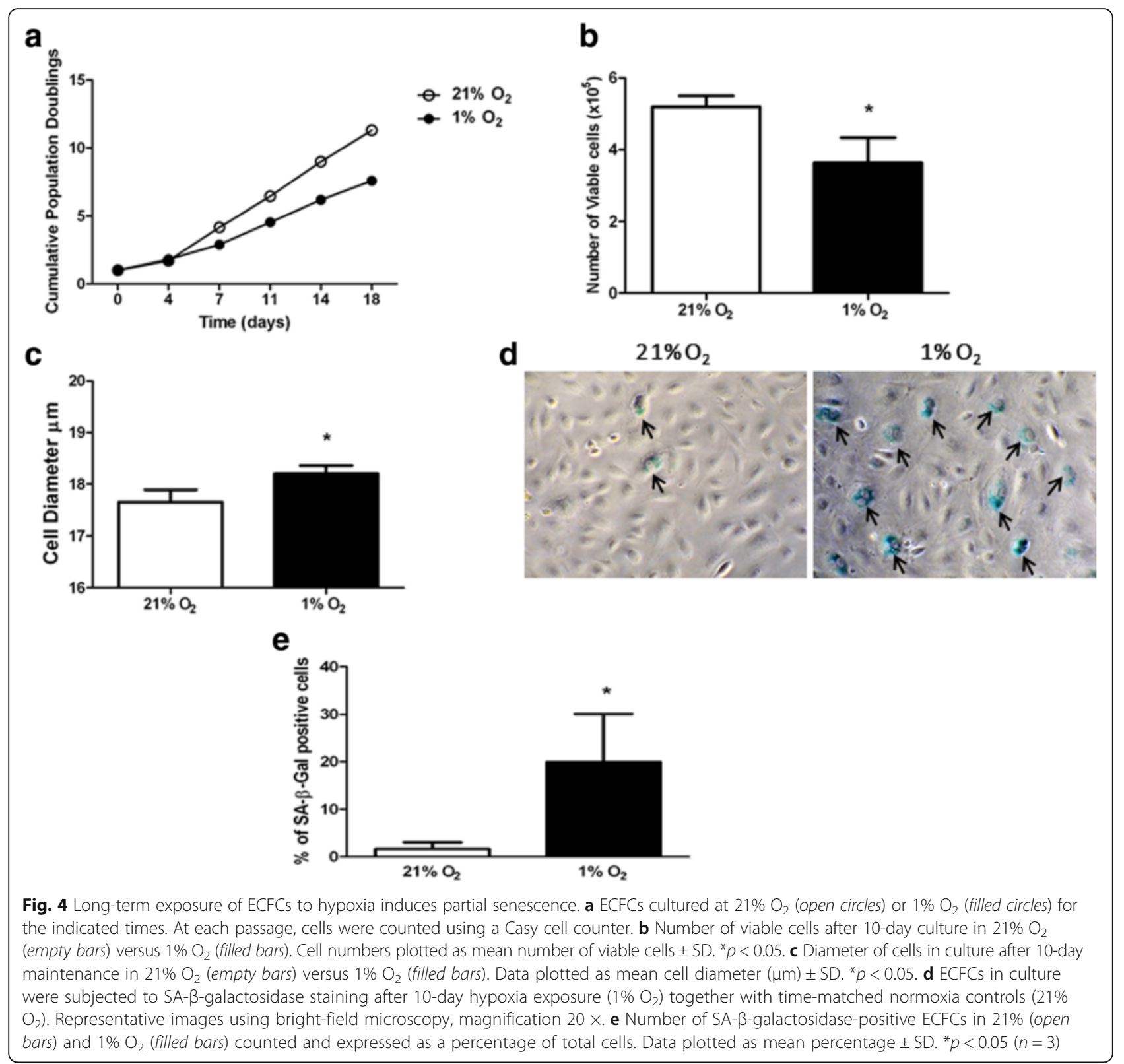

to the surface of the culture flasks. To further explore the effect of low oxygen on ECFC phenotype, cells were maintained in $1 \% \mathrm{O}_{2}$ for up to 18 days. Chronic hypoxia reduced ECFC cell doubling and viability (Fig. 4a, b). Consistent with the cytoskeletal changes seen after $48 \mathrm{~h}$, ECFC cell size increased after culture in $1 \% \mathrm{O}_{2}$ for 10 days (Fig. 4c). The number of $\beta$-galactosidase-positive ECFCs increased significantly after long-term culture in $1 \% \mathrm{O}_{2}$, suggestive of a senescence-like phenotype in a proportion of these cells (Fig. 4d, e). Together, these data suggest that hypoxia induces changes in ECFC phenotype suggestive of a more adhesive, less mitotic, senescent-like phenotype. To test this hypothesis, scratch wound assays were carried out at 21 and $1 \% \mathrm{O}_{2}$. Exposure of ECFCs to $1 \% \mathrm{O}_{2}$ decreased the wound closure rate compared with cells grown in $21 \% \mathrm{O}_{2}$, although this difference was not significant (Fig. 5a, b). Thus, changes in ECFC cytoskeleton are consistent with an increased adhesive capacity linked to a reduced migratory potential in ECFCs.

ECFCs are believed to be recruited from a defined niche into the systemic circulation, after which they home to an area of tissue ischaemia/hypoxia where they stimulate vascular repair and/or new blood vessel formation [26]. In the in-vitro setting, tubulogenesis in Matrigel $^{\circ}$ is a robust indicator of vasculogenic function of ECFCs growing under normal atmospheric oxygen. Incubation of ECFCs under normal oxygen conditions triggered tube formation that was evident at $48 \mathrm{~h}$ (Fig. 6a, b). In contrast, tube area 


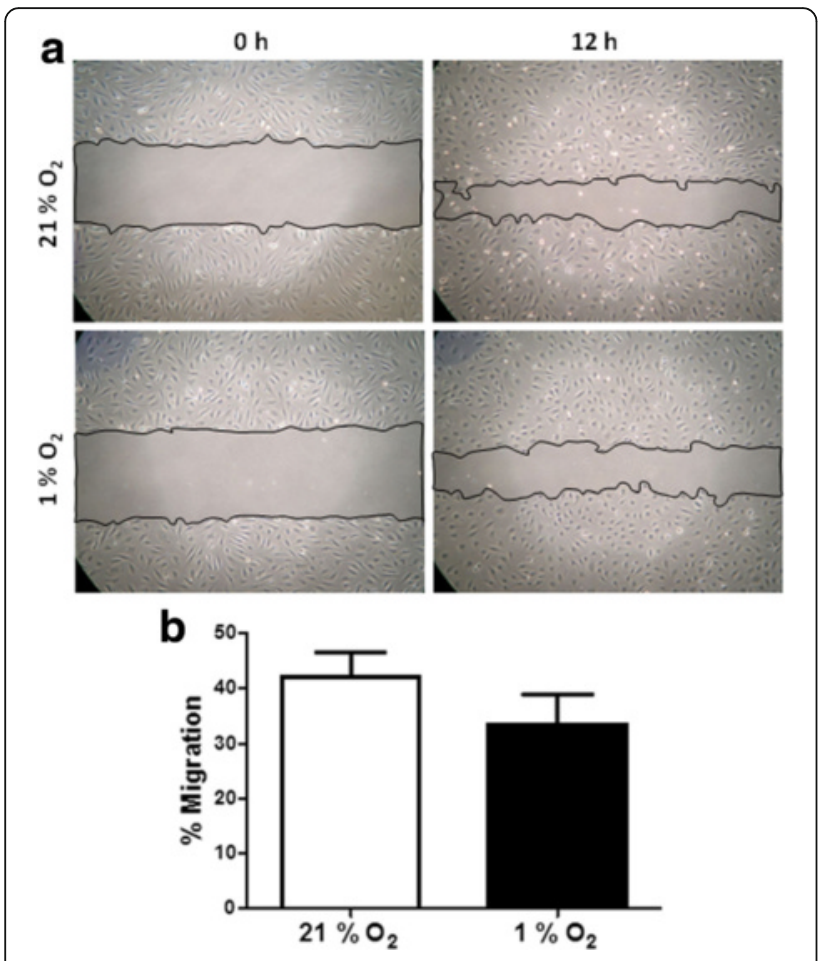

Fig. 5 Hypoxia delays scratch wound repair and tube formation in ECFCs. The ECFCs were grown in 21 and $1 \% \mathrm{O}_{2}$. Cells were photographed at the time of scratch and at $12 \mathrm{~h}$ post wounding. a Representative images of scratch wound at time zero $(0 h)$ and 12 hours $(12 \mathrm{~h})$. Bars indicate the boundaries of the scratch measured at each time point. b Percentage migration 12-h post scratch. Each condition was compared separately and the percentage migration calculated for $21 \% \mathrm{O}_{2}$ (empty bars) or $1 \% \mathrm{O}_{2}$ (filled bars). Data plotted as mean \pm SEM of three independent experiments $(n=4)$

was significantly lower in ECFCs cultured in $1 \% \mathrm{O}_{2}$. These data suggest that hypoxia decreases ECFC-mediated tube formation in vitro.

ECFCs are known to secrete a range of growth factors and cytokines which have autocrine stimulatory function [27], although it is unknown how exposure to hypoxia influences this secretome. A profile of secreted angiogenic factors from the conditioned medium of ECFCs grown in 21 and $1 \% \mathrm{O}_{2}$ for $24 \mathrm{~h}$ was identified using a Proteome Profiler (R\&D Systems). When compared with ECFCs growing in normal atmospheric oxygen condition, hypoxia-exposed cells secreted higher levels of VEGF and PlGF (Fig. 7a-c). Consistently, levels of PIGF were significantly increased at $48 \mathrm{~h}$ when the conditioned medium of ECFCs grown in $1 \%$ versus $21 \% \mathrm{O}_{2}$ was analysed by ELISA (Fig. 7d). Importantly, increased levels of PIGF were maintained in ECFCs exposed to $1 \%$ $\mathrm{O}_{2}$ for 11 days, suggesting that PlGF secretion is not only a short-term response to low oxygen, but is also maintained during chronic exposure to hypoxia, which is suggestive of an adaptive response of these cells to a hypoxic environment (Fig. 7e).
To establish whether this upregulation of PlGF occurred as a result of hypoxia-induced stabilisation of HIF- $1 \alpha$ and other transcription factors, ECFCs were treated with dimethyloxalylglycine (DMOG), a cellpermeable inhibitor of prolyl hydroxylases (PHDs) that decreases proteosomal-mediated destruction of HIF- $1 \alpha$. Stabilisation of HIF- $1 \alpha$ was observed in ECFCs grown in $21 \% \mathrm{O}_{2}$ treated with DMOG for $24 \mathrm{~h}$ (Fig. 8a). Conditioned medium from DMOG-treated ECFCs also contained higher levels of PIGF, with a higher foldchange than that observed in ECFCs incubated in $1 \%$ $\mathrm{O}_{2}$ versus $21 \% \mathrm{O}_{2}$ (Fig. 8b). Consistent with previous data (Figs. 4b and 6), conditioned medium from DMOG-treated ECFCs decreased tube formation and cell viability in ECFCs, mimicking the effect seen in $1 \% \mathrm{O}_{2}$ (Fig. 8c, d).

To test whether PIGF in the conditioned medium from ECFCs was mediating this inhibition of tube formation, a neutralising PlGF antibody was used to inhibit PlGF in these experiments. Exposure of ECFCs to conditioned medium from DMOG-treated cells inhibited tube formation, as seen previously (Fig. 9, a versus b). Significantly, incubation of conditioned medium from DMOG-treated ECFCs with anti-PIGF neutralising antibody reversed this inhibitory effect and returned tube formation to control levels (Fig. 9, c versus d, e). The inclusion of an isotype antibody control in these experiments suggests that this effect is specifically due to the binding and inhibition of PlGF by the neutralising antibody (Fig. 9c).

\section{Discussion}

ECFCs remain a promising candidate for cell therapy to treat tissue ischaemia. However, robust characterisation of ECFC cell behaviour in the target tissues and diseaserelated microenvironments is required as these cells move towards therapy. In this study, we have defined the response of ECFCs to hypoxia exposure, one of the main factors associated with vascular insufficiency and tissue ischaemia. The HIF transcription factors are considered master regulators of hypoxia, having an established role in the cellular response to oxygen deprivation, and are of critical importance in hypoxia-driven angiogenesis by controlling the expression of many genes required for the process [28]. In response to a graded decrease in $\mathrm{O}_{2}$, HIF- $1 \alpha$ stabilisation was detected in ECFCs at the lower $\mathrm{O}_{2}$ levels of 3 and $1 \%$ with no HIF stabilisation detectable at the higher oxygen concentrations of 5 and $10 \%$ (Fig. 1). Similarly, in a study investigating the effect of low oxygen levels on ECFCs and MSCs, Hofmann et al. [29] reported that while MSCs and fibroblasts are very sensitive to lowering oxygen concentration HIF- $1 \alpha$ stabilisation only occurred at $1 \% \mathrm{O}_{2}$ levels. Accumulation of HIF-1 $\alpha$ occurred within $5 \mathrm{~min}$ in these cells (Fig. 1b). These findings are important because the physiological oxygen tension varies widely between different cell types 


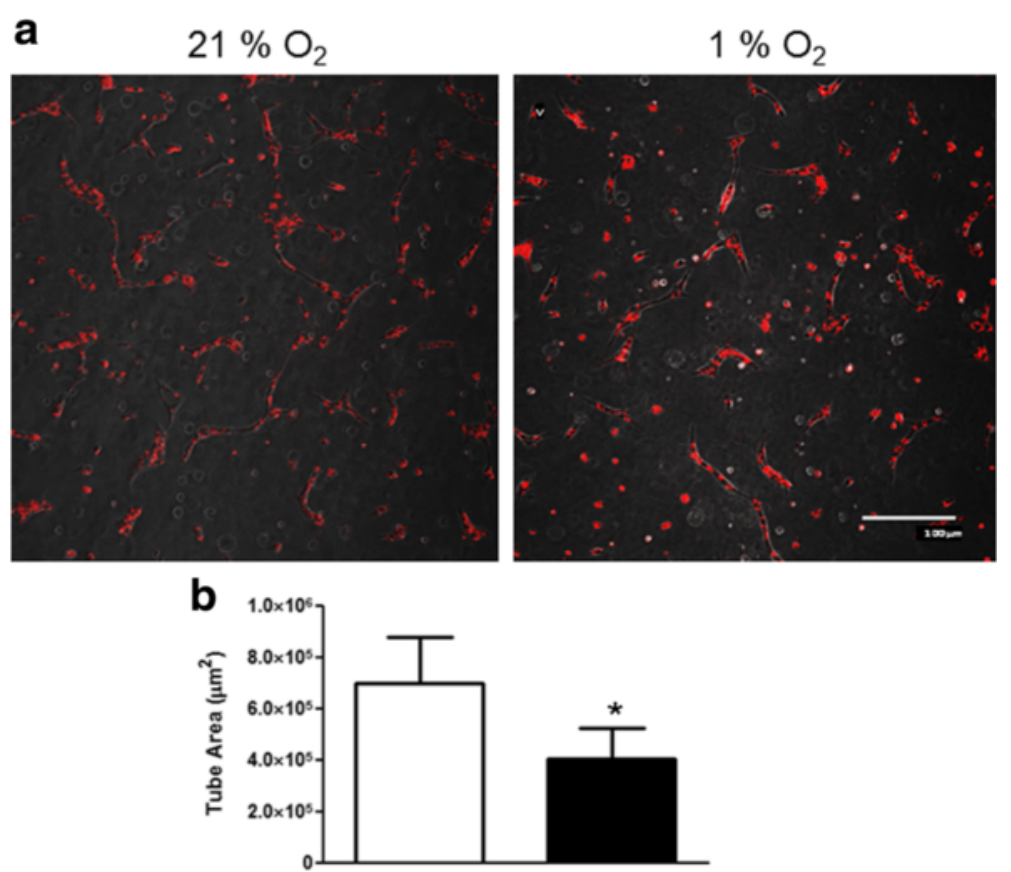

Fig. 6 Hypoxia exposure decreases ECFC angiogenesis in vitro. a ECFCs were stained with PKH membrane dye and grown in Matrige ${ }^{\circledR}$, and the formation of tube-like structures was assessed after $48 \mathrm{~h}$. Representative images for 21 and $1 \% \mathrm{O}_{2}$ are shown. Scale bar: $100 \mu \mathrm{m}$. b Total tube area $\left(\mu \mathrm{m}^{2}\right.$ ) was quantified using NIS Elements software (Nikon) for ECFCs grown in $21 \% \mathrm{O}_{2}$ (empty bars) or $1 \% \mathrm{O}_{2}$ (filled bars). Data plotted as mean \pm SD. ${ }^{*} p<0.05$. Scale bars: $100 \mu \mathrm{m}(n=3)$

and tissues. For example, alveoli have a physiological oxygen concentration of approximately $14.5 \%$ (approximately $110 \mathrm{mmHg}$ ), while the brain has a much lower physiological oxygen percentage in the region of $4.4 \%$ (34 $\mathrm{mmHg})$ [30]. In fact, culturing ECFCs in ambient $21 \% \mathrm{O}_{2}$ may actually elevate the physiological oxygen tension above baseline, where key stem cell niches such as the bone marrow would normally experience a much lower physiological oxygen tension of around $6.4 \%$ (49 $\mathrm{mmHg}$ ) [30]. Other groups have found that culturing induced pluripotent stem cells (iPS) and mesenchymal stem cells (MSCs) at low oxygen concentrations provides enhanced functional outcomes [31,32]. While not within the remit of this study, future studies will probably define the optimum oxygen concentration for ex-vivo ECFC culture. Maintenance in an oxygen concentration likely to be experienced in vivo may improve the therapeutic potential of these cells for clinical use. For this study, we chose $1 \%$ $\mathrm{O}_{2}$ based on robust stabilisation of HIF- $1 \alpha$ and HIF $2 \alpha$, along with HIF-1 $\alpha$ nuclear translocation and hypoxiadependent gene expression changes that were detected under these conditions (Figs. 1 and 2).

ECFCs cultured in $1 \% \mathrm{O}_{2}$ for $24-72 \mathrm{~h}$ displayed changes in their actin cytoskeleton (Fig. 3), as well as cell doubling, increased cell size and staining for senescence markers (Fig. 4). Increased filamentous actin, along with the appearance of focal adhesions at the end of actin stress fibres, suggested that hypoxia was inducing enhanced
ECFC adherence to the cell culture dish (Fig. 3). One interpretation of these data is that when ECFCs are recruited to the ischaemic/hypoxic tissue, they need to attach to the extracellular matrix in close proximity to either damaged blood vessels or avascular regions. These data were supported by reduced ECFC scratch wound repair in $1 \% \mathrm{O}_{2}$ versus $21 \% \mathrm{O}_{2}$ (Fig. 5). Reductions in ECFC chemotaxis/migration in response to low oxygen have been reported by both the Yoder and Strunk groups [29, 33], while the Yoder group also detected an increase in hypoxia-mediated tube formation in ECFCs, albeit at an earlier time point than our study $(6 \mathrm{~h}$ versus $72 \mathrm{~h}$, Fig. $4 \mathrm{~d}$ [33]). Hofmann et al. [29] also report a decrease in tube formation in ECFCs when exposed to $1 \% \mathrm{O}_{2}$. In vascular smooth muscle cells, HIF- $1 \alpha$ expression decreased cell migration and adherence to the ECM, with no evident changes in vincullin or focal adhesion kinase (FAK) localisation [34]. Hypoxia increased MSC migration, probably via VEGF-mediated phosphorylation of FAK and activation of MAPK and eNOS signalling [35]. Hypoxia increased $\alpha v \beta 3$ integrin cell surface expression and cell adhesion in trophoblast stem cells [36]. Together with our results, these data suggest that there may be cell-type specific responses to low oxygen, with ECFCs demonstrating increased adhesiveness and decreased proliferation and migration, perhaps to ensure their effective engraftment to the ischaemic tissue in vivo. Consistently, we have identified that exposure of ECFCs to hypoxia decreases the 


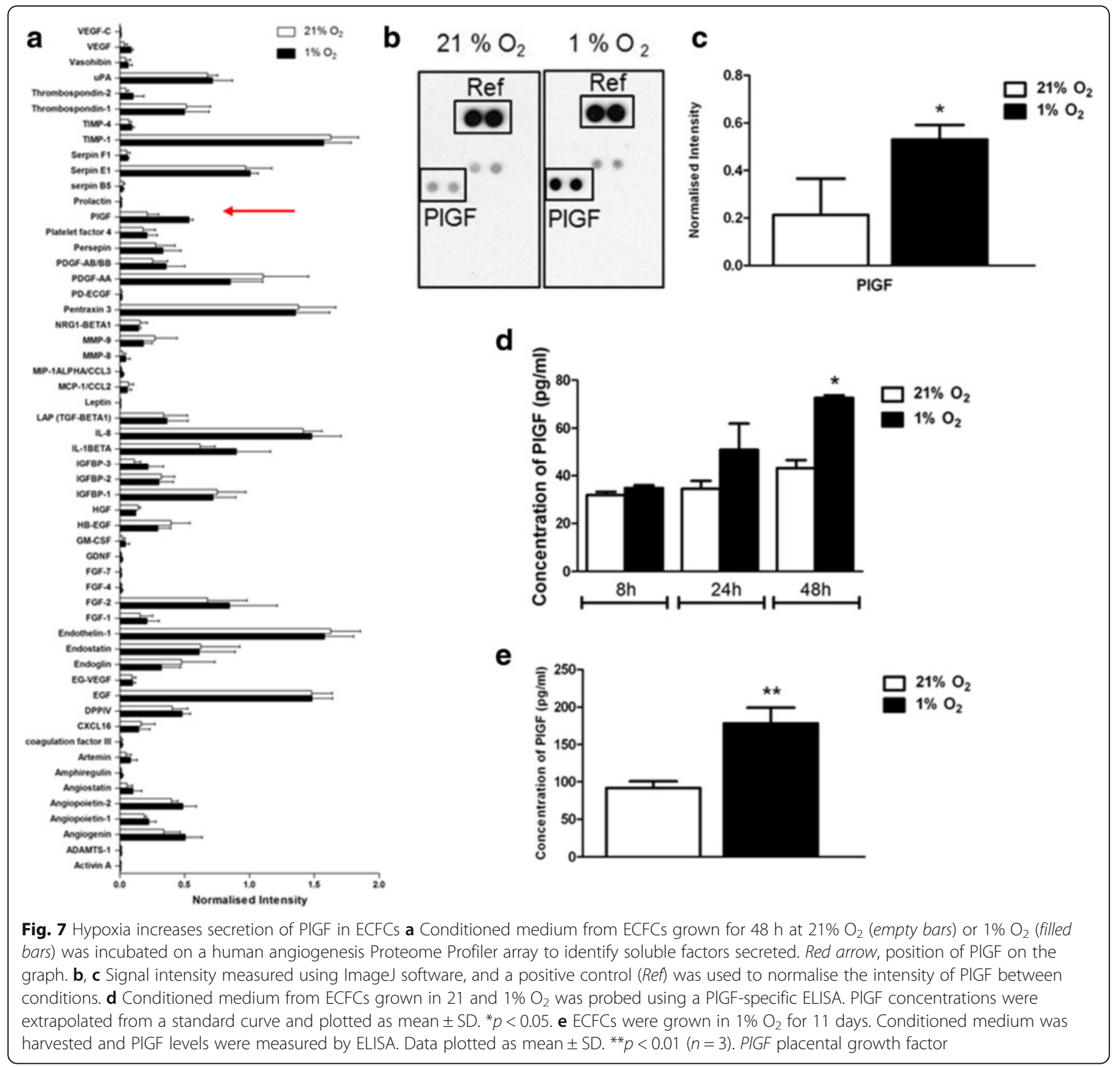

expression of CD34, a marker of "stemness", which suggests that ECFCs in the hypoxic niche become more endothelial-like to perhaps improve their ability to repair damaged endothelium (data not shown).

One of the key findings of this study was the increased secretion of PlGF by ECFCs (Fig. 6). Despite the robust increase in PIGF protein expression in $1 \% \mathrm{O}_{2}$ or in response to DMOG, an inhibitor of HIF prolyl hydroxylases [37], previous analysis of the PlGF promoter and enhancer regions did not identify any the putative binding sites for HIFs (hypoxia response elements (HREs)) [38-40]. Consistent with data from Xiang et al. [41], we did not detect any increase in PIGF RNA in hypoxia-exposed ECFCs. In contrast, overexpression of HIF- $1 \alpha$ has been reported by others to augment the expression of PlGF in both endothelial cells [42-44] and primary cardiac and vascular cells [45], indicating that HIFs may well have a role to play in PIGF expression. A recent report identified novel HIF- $1 \alpha$ HREs in intron 2 of the PlGF gene, and highlighted chromatin remodelling via histone $\mathrm{H} 3$ and H4 acetylation as a key element in HIF $1 \alpha$-mediated PIGF upregulation $[44,46]$. Other transcription factors such as NFKB and CREB are also stabilised under low oxygen conditions, and may play a role in PlGF upregulation [41, 42, 45, 46]. PlGF has been reported to enhance angiogenic signalling by acting synergistically with VEGF to increase signalling via the receptor VEGFR2 $[47,48]$ while also maintaining the ability to 
a
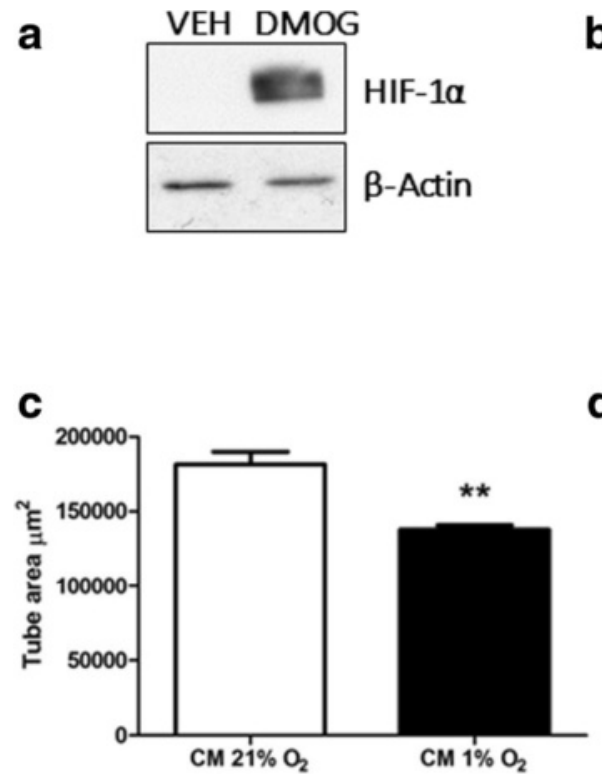

b

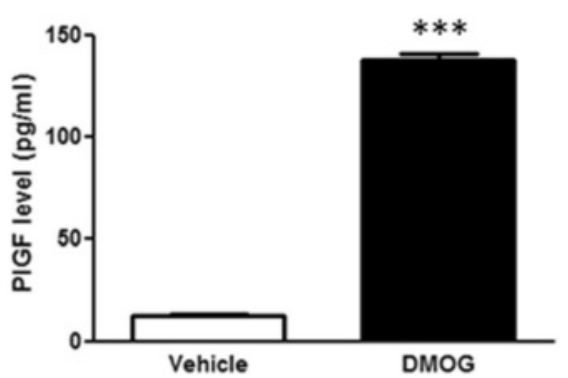

d

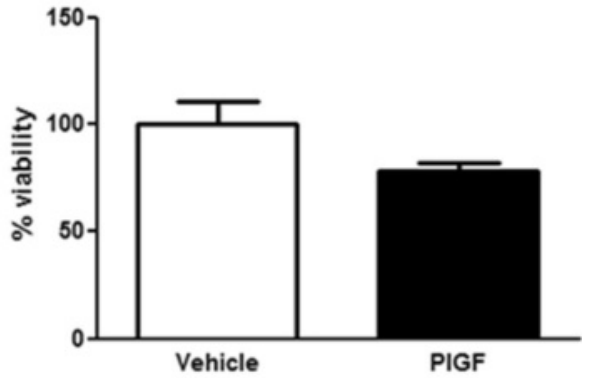

Fig. 8 HIF-1a stabilisation induces PIGF expression and alters ECFC tube formation and viability. a ECFCs were grown in Matrigel ${ }^{\circledR}$ and exposed to vehicle (DMSO, VEH) or DMOG (1 mM) for $24 \mathrm{~h}$. Protein lysates were probed for HIF1a (upper panel) or $\beta$-actin (lower panel). b PIGF concentration was measured by ELISA in conditioned medium from ECFCs treated with vehicle (DMSO) or DMOG (1 mM) for 24 h. c Conditioned medium (CM)from ECFCs treated with vehicle (DMSO) or DMOG (1 mM) was used to treat ECFCs grown in Matrige ${ }^{\circledR}$ and stained with PKH for $24 \mathrm{~h}$. Total tube area $\left(\mu \mathrm{m}^{2}\right)$ quantified using NIS Elements software. $\mathbf{d}$ ECFCs were treated with conditioned medium from vehicle (DMSO) or DMOG (1 mM)treated cells for $24 \mathrm{~h}$. Cell viability measured using a CCK-8 cell viability assay (Sigma), and plotted as mean \% viability \pm SD $(n=4)$. DMOG dimethyloxalylglycine, PIGF placental growth factor. Data plotted as Mean \pm SD ${ }^{* *} p<0.01 ;{ }^{* * *} p<0.001$
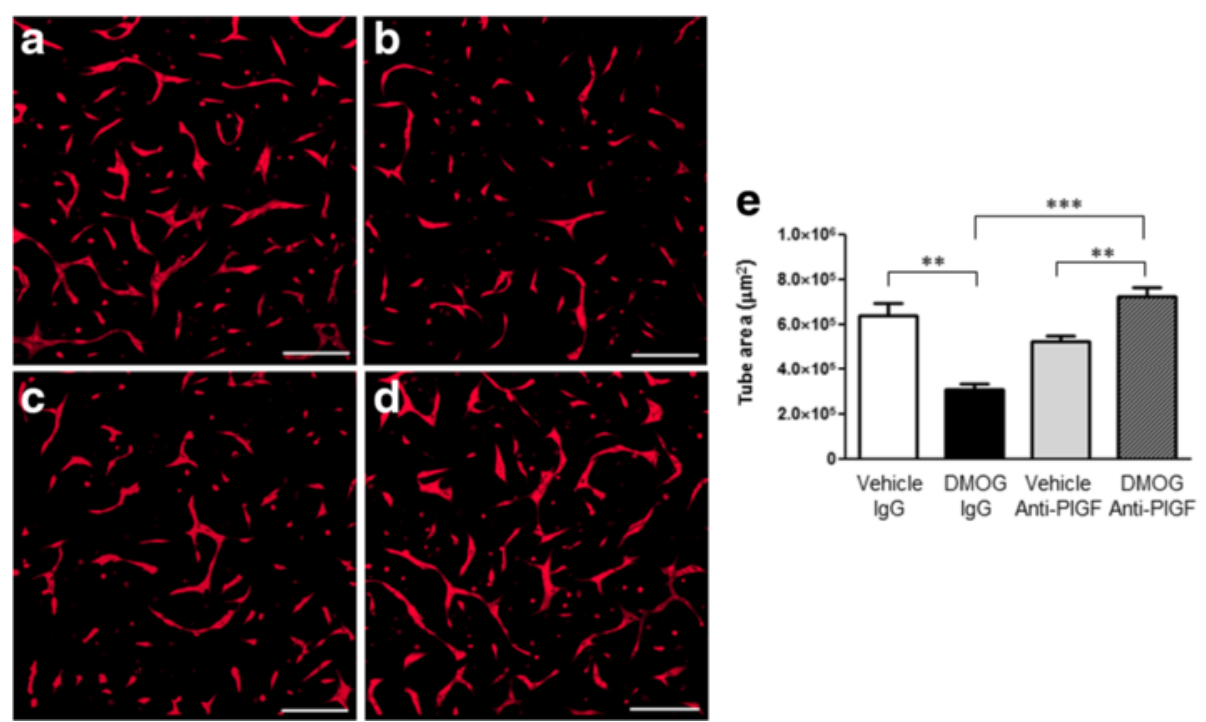

Fig. 9 Anti-PIGF neutralising antibody blocks DMOG-induced inhibition of ECFC tube formation. ECFCs were stained with calcein (Invitrogen) and grown on Matrigel ${ }^{\oplus}$. Cells were treated with conditioned medium harvested from ECFCs treated with a DMSO (vehicle) plus isotype control lgG, b DMOG plus isotype control lgG, c DMSO plus anti-PIGF neutralising antibody or d DMOG plus anti-PIGF neutralising antibody. e At 48 h, total tube area $\left(\mu \mathrm{m}^{2}\right)$ was quantified using NIS Elements software (Nikon). Data plotted as mean \pm SD and are representative of three independent experiments. ${ }^{* *} p<0.01 ;{ }^{* *} p<0.001$. Scale bars: $200 \mu \mathrm{m}(n=3)$. DMOG dimethyloxalylglycine, PIGF placental growth factor 
induce angiogenic signalling independent of VEGF [49]. Others have identified that PIGF may increase blood vessel formation via monocyte recruitment and paracrine signalling to endothelial cells [41, 44]. Increased PIGF secretion from ECFCs coincided with a delay in in-vitro tube formation in Matrigel $^{\circ}$ (Fig. 8b, c). Neutralising antibodies against PlGF rescue ECFC tube formation in Matrigel $^{\circ}$ (Fig. 9). Thus, hypoxia/HIF-mediated increases in PlGF secretion from ECFCs may inhibit tube formation and vasculogenesis.

Our data suggest that, in vitro, in the absence of VEGF, PIGF may not be driving ECFC tube formation, and heterodimers of PIGF and VEGF may be the active moieties. This is somewhat surprising, given the previously described pro-angiogenic activity of PIGF [50]. It remains to be determined whether PlGF released from ECFCs in the hypoxic niche would have both an autocrine and a paracrine function, and what the relative balance of these functions might be. We can also speculate that PIGF from chronically hypoxia-exposed ECFCs may function to attract monocytes, pericytes or mural cells to stabilise the vascular niche. PIGF may also be secreted from the cells of the tissue parenchyma in ischaemic tissue. Alternatively, a paracrine source of VEGF or other factor from cells such as pericytes or endothelial cells may be required to trigger new blood vessel formation. ECFCs have been demonstrated to act as paracrine mediators, modulating MSC regeneration potential prior to the establishment of neovascularisation and blood perfusion, ultimately enabling extensive engraftment and long-term differentiation of transplanted MSCs [51]. Recent work by Xiang et al. [41, 44] supports the idea of a paracrine role for PIGF secreted by endothelial cells in vascular repair.

\section{Conclusions}

This study deepens our understanding of the response of ECFCs to hypoxia and also addresses important questions regarding the role of PlGF and chronic hypoxia in influencing the neovascular potential of ECFCs. Further studies will be required to determine whether ex-vivo pre-conditioning of ECFCs, by either hypoxia or PlGF, or manipulation of PlGF expression in vivo may be useful tools to augment ECFC neovascularisation for the future treatment of ischaemic disease.

\section{Additional file}

\footnotetext{
Additional file 1: Figure S1. showing characterisation of ECFC identity using flow cytometry. ECFCs were grown in complete EBM2 medium at $21 \% \mathrm{O}_{2}$. Cells were trypsinised and stained with antibodies against endothelial markers CD31 and CD146 (green) and hematopoietic markers CD45 and CD14 (red). Respective isotype controls are shown in black and $\%$ positivity is shown in the bottom right-hand corner. Cell number plotted on $x$ axis and fluorescence intensity plotted on $Y$ axis. Data are
}

representative of experiments carried out on at least seven ECFC clones $(n=7)$. (TIF $73 \mathrm{~kb})$

\section{Abbreviations}

ANGPTL4: Angiopoietin-like 4; CREB: cAMP response element-binding protein; DMOG: Dimethyloxalylglycine; ECFC: Endothelial colony-forming cell; eNOS: Endothelial nitric oxide synthase; EPC: Endothelial progenitor cell; FAK: Focal adhesion kinase; GLUT1: Glucose transporter 1; HIF-1a: Hypoxia inducible factor 1 alpha; HIF-2a: Hypoxia inducible factor 2 alpha; MAPK: Mitogen-activated protein kinase; MUC18: Melanoma metastasisassociated surface molecule; NFKB: Nuclear factor kappa-light-chain-enhancer of activated B cells; OEC: Outgrowth endothelial cell; PECAM: Platelet endothelial cell adhesion molecule; PIGF: Placental growth factor;

SLC2A1: Solute carrier family 2, facilitated glucose transporter member 1; VEGF: Vascular endothelial growth factor; VEGFR2: Vascular endothelial growth factor receptor 2

\section{Acknowledgements}

The authors thank Lynsey-Dawn Allen and other technical staff at the Centre of Experimental Medicine for technical assistance.

\section{Funding}

This work was supported by Action Medical Research UK. Work in the laboratory of Derek Brazil is supported by the Wellcome Trust, Northern Ireland Kidney Research Fund and DEL NI.

\section{Availability of data and materials}

The datasets for and/or analysed during the current study are available from the corresponding author on reasonable request.

\section{Authors' contributions}

$\mathrm{MBH}$ was responsible for conception and design, collection and assembly of data, data analysis and interpretation, and manuscript writing. IHAA was responsible for collection and assembly of data, data analysis and interpretation. CLO, EH, MHL and TS were responsible for collection and assembly of data. RM, SC, BR, EM, DS and AWS were responsible for provision of study material or patients. DPB was responsible for conception and design, data analysis and interpretation, and manuscript writing. All authors have been involved in revising and approving the final manuscript.

\section{Competing interests}

The authors declare that they have no competing interests.

\section{Consent for publication}

Not applicable.

Ethical approval and consent to participate

The Office for Research Ethics Committees Northern Ireland gave full approval for this study (08/NIR02/02). All participants gave written and informed consent prior to collection of blood from umbilical cords.

\section{Author details}

${ }^{1}$ Centre for Experimental Medicine, Queen's University Belfast, 97 Lisburn Road, Belfast BT9 7BL, UK. Eye \& Ear Clinic, Royal Victoria Hospital, Grosvenor Road, Belfast BT12 6BA, UK. ${ }^{3}$ Regional Neonatal Unit, Royal Maternity Hospital, Grosvenor Road, Belfast BT12 6BA, UK.

Received: 16 July 2016 Revised: 10 October 2016 Accepted: 26 October 2016 Published online: 29 November 2016

\section{References}

1. Asahara T, Murohara T, Sullivan A, et al. Isolation of putative progenitor endothelial cells for angiogenesis. Science. 1997;275:964-7.

2. Yoder MC, Mead LE, Prater D, et al. Redefining endothelial progenitor cells via clonal analysis and hematopoietic stem/progenitor cell principals. Blood. 2007;109:1801-9.

3. Medina RJ, O'Neill CL, O'Doherty TM, et al. Endothelial progenitors as tools to study vascular disease. Stem Cells Int. 2012;2012:346735. 
4. Medina RJ, O'Neill CL, Humphreys MW, et al. Outgrowth endothelial cells: characterization and their potential for reversing ischemic retinopathy. Invest Ophthalmol Vis Sci. 2010;51:5906-13.

5. Lois N, McCarter RV, O'Neill C, et al. Endothelial progenitor cells in diabetic retinopathy. Front Endocrinol (Lausanne). 2014;5:44.

6. Fadini GP, Avogaro A. It is all in the blood: the multifaceted contribution of circulating progenitor cells in diabetic complications. Exp Diabetes Res. 2012;2012:742976.

7. Jarajapu YP, Hazra S, Segal M, et al. Vasoreparative dysfunction of CD34+ cells in diabetic individuals involves hypoxic desensitization and impaired autocrine/paracrine mechanisms. PLoS One. 2014;9:e93965.

8. Chan $\mathrm{KH}$, Simpson PJ, Yong AS, et al. The relationship between endothelial progenitor cell populations and epicardial and microvascular coronary disease-a cellular, angiographic and physiologic study. PLoS One. 2014:9:e93980.

9. Muñoz-Hernandez R, Miranda ML, Stiefel P, et al. Decreased level of cord blood circulating endothelial colony-forming cells in preeclampsia. Hypertension. 2014;64:165-71.

10. Safranow K, Kotowski M, Lewandowska J, et al. Circulating endothelial progenitor cells in premature infants: is there an association with premature birth complications? J Perinat Med. 2012;40:455-62.

11. Ligi I, Simoncini S, Tellier E, et al. A switch toward angiostatic gene expression impairs the angiogenic properties of endothelial progenitor cells in low birth weight preterm infants. Blood. 2011;118:1699-709.

12. Vassallo PF, Simoncini S, Ligi I, et al. Accelerated senescence of cord blood endothelial progenitor cells in premature neonates is driven by SIRT1 decreased expression. Blood. 2014;123:2116-26.

13. De Falco $\mathrm{S}$. The discovery of placenta growth factor and its biological activity. Exp Mol Med. 2012;44:1-9.

14. Roskoski Jr R. VEGF receptor protein-tyrosine kinases: structure and regulation. Biochem Biophys Res Commun. 2008;375:287-91.

15. Maglione D, Guerriero V, Viglietto $G$, et al. Isolation of a human placenta cDNA coding for a protein related to the vascular permeability factor. Proc Natl Acad Sci U S A. 1991;88:9267-71.

16. Dewerchin M, Carmeliet P. PIGF: a multitasking cytokine with diseaserestricted activity. Cold Spring Harb Perspect Med. 2012;2:901156.

17. Avouac J, Meune C, Ruiz B, et al. Angiogenic biomarkers predict the occurrence of digital ulcers in systemic sclerosis. Ann Rheum Dis. 2012;71:394-9.

18. d'Audigier C, Gautier B, Yon A, et al. Targeting VEGFR1 on endothelial progenitors modulates their differentiation potential. Angiogenesis. 2014;17:603-16

19. Li B, Wang C, Zhang Y, et al. Elevated PLGF contributes to small-cell lung cancer brain metastasis. Oncogene. 2013;32:2952-62.

20. Park SJ, Kim KJ, Kim WU, et al. Interaction of mesenchymal stem cells with fibroblast-like synoviocytes via cadherin-11 promotes angiogenesis by enhanced secretion of placental growth factor. J Immunol. 2014;192:3003-10.

21. Chappell LC, Duckworth S, Seed PT, et al. Diagnostic accuracy of placental growth factor in women with suspected preeclampsia: a prospective multicenter study. Circulation. 2013:128:2121-31.

22. Zhao J, Bolton EM, Randle $L$, et al. Functional characterization of late outgrowth endothelial progenitor cells in patients with end-stage renal failure. Transpl Int. 2014;27:437-51.

23. Weidt C, Niggemann B, Kasenda B, et al. Stem cell migration: a quintessential stepping stone to successful therapy. Curr Stem Cell Res Ther. 2007;2:89-103.

24. Medina RJ, O'Neill CL, Sweeney M, et al. Molecular analysis of endothelial progenitor cell (EPC) subtypes reveals two distinct cell populations with different identities. BMC Med Genomics. 2010;3:18.

25. Ceradini DJ, Gurtner GC. Homing to hypoxia: HIF-1 as a mediator of progenitor cell recruitment to injured tissue. Trends Cardiovasc Med. 2005;15:57-63.

26. Bouvard C, Gafsou B, Dizier B, et al. alpha6-integrin subunit plays a major role in the proangiogenic properties of endothelial progenitor cells. Arterioscler Thromb Vasc Biol. 2010;30:1569-75.

27. Medina RJ, O'Neill CL, O'Doherty TM, et al. Ex vivo expansion of human outgrowth endothelial cells leads to IL-8-mediated replicative senescence and impaired vasoreparative function. Stem Cells. 2013;31:1657-68.

28. Pugh CW, Ratcliffe PJ. Regulation of angiogenesis by hypoxia: role of the HIF system. Nat Med. 2003;9:677-84

29. Hofmann NA, Ortner A, Jacamo RO, et al. Oxygen sensing mesenchymal progenitors promote neo-vasculogenesis in a humanized mouse model in vivo. PLoS One. 2012;7:e44468.
30. Koh MY, Powis G. Passing the baton: the HIF switch. Trends Biochem Sci. 2012;37:364-72

31. Liu SP, Li YX, Xu J, et al. An improved method for generating integrationfree human induced pluripotent stem cells. J Exp Hematol. 2014;22:580-7.

32. Saller MM, Prall WC, Docheva D, et al. Increased stemness and migration of human mesenchymal stem cells in hypoxia is associated with altered integrin expression. Biochem Biophys Res Commun. 2012;423:379-85.

33. Decaris $\mathrm{ML}$, Lee $\mathrm{Cl}$, Yoder $\mathrm{MC}$, et al. Influence of the oxygen microenvironment on the proangiogenic potential of human endothelial colony forming cells. Angiogenesis. 2009;12:303-11.

34. Corley KM, Taylor CJ, Lilly B. Hypoxia-inducible factor 1alpha modulates adhesion, migration, and FAK phosphorylation in vascular smooth muscle cells. J Cell Biochem. 2005;96:971-85.

35. Lee SH, Lee YJ, Song CH, et al. Role of FAK phosphorylation in hypoxiainduced hMSCS migration: involvement of VEGF as well as MAPKS and eNOS pathways. Am J Physiol Cell Physiol. 2010;298:C847-56.

36. Cowden Dahl KD, Fryer BH, Mack FA, et al. Hypoxia-inducible factors 1alpha and 2alpha regulate trophoblast differentiation. Mol Cell Biol. 2005;25:10479-91.

37. Cummins EP, Seeballuck F, Keely SJ, et al. The hydroxylase inhibitor dimethyloxalylglycine is protective in a murine model of colitis. Gastroenterology. 2008;134:156-65.

38. Green CJ, Lichtlen P, Huynh NT, et al. Placenta growth factor gene expression is induced by hypoxia in fibroblasts: a central role for metal transcription factor-1. Cancer Res. 2001;61:2696-703.

39. Oura H, Bertoncini J, Velasco P, et al. A critical role of placental growth factor in the induction of inflammation and edema formation. Blood. 2003;101:560-7.

40. Selvaraj SK, Giri RK, Perelman N, et al. Mechanism of monocyte activation and expression of proinflammatory cytochemokines by placenta growth factor. Blood. 2003;102:1515-24.

41. Xiang L, Varshney R, Rashdan NA, et al. Placenta growth factor and vascular endothelial growth factor-A have differential, cell-type specific patterns of expression in vascular cells. Microcirculation. 2014;21(5):368-79.

42. Lappas M. Nuclear factor-kappaB mediates placental growth factor induced pro-labour mediators in human placenta. Mol Hum Reprod. 2012:18:354-61.

43. Depoix C, Tee MK, Taylor RN. Molecular regulation of human placental growth factor (PIGF) gene expression in placental villi and trophoblast cells is mediated via the protein kinase a pathway. Reprod Sci. 2011;18:219-28.

44. Yamakawa M, Liu LX, Date T, et al. Hypoxia-inducible factor-1 mediates activation of cultured vascular endothelial cells by inducing multiple angiogenic factors. Circ Res. 2003;93:664-73.

45. Kelly BD, Hackett SF, Hirota K, et al. Cell type-specific regulation of angiogenic growth factor gene expression and induction of angiogenesis in nonischemic tissue by a constitutively active form of hypoxia-inducible factor 1. Circ Res. 2003;93:1074-81.

46. Tudisco L, Della Ragione F, Tarallo V, et al. Epigenetic control of hypoxia inducible factor-1alpha-dependent expression of placental growth factor in hypoxic conditions. Epigenetics. 2014;9:600-10.

47. Carmeliet $P$, Moons L, Luttun A, et al. Synergism between vascular endothelial growth factor and placental growth factor contributes to angiogenesis and plasma extravasation in pathological conditions. Nat Med. 2001;7:575-83.

48. Autiero M, Waltenberger J, Communi D, et al. Role of PIGF in the intra- and intermolecular cross talk between the VEGF receptors Flt1 and Flk1. Nat Med. 2003:9:936-43.

49. Holmes DI, Zachary I. Placental growth factor induces FosB and c-Fos gene expression via Flt-1 receptors. FEBS Lett. 2004;557:93-8.

50. Iwasaki H, Kawamoto A, Tjwa M, et al. PIGF repairs myocardial ischemia through mechanisms of angiogenesis, cardioprotection and recruitment of myo-angiogenic competent marrow progenitors. PLoS One. 2011;6:e24872.

51. Lin RZ, Moreno-Luna R, Li D, et al. Human endothelial colony-forming cells serve as trophic mediators for mesenchymal stem cell engraftment via paracrine signaling. Proc Natl Acad Sci U S A. 2014;111:10137-42. 